\title{
Facial Swelling and Shortness of Breath Are Not Always an Allergic Reaction: It Could Be Acute Superior Vena Cava Syndrome
}

\author{
Rana Al-Zakharia, d, Basma Ataallah ${ }^{\mathrm{b}}$, Harith Alataby ${ }^{\mathrm{a}}$, Jay Nfonoyimª, c
}

\begin{abstract}
Historically, it has been found that malignancy is associated with superior vena cava (SVC) syndrome. The past decade has seen more cases of thrombogenic and stenotic SVC syndrome due to increased use of pacemakers and indwelling central lines. As compared to the slowly progressing obstruction in malignancy, rapid thrombogenesis rate and a lack of venous collateral sequelae lead to more acute sequelae in these patients. It is important to timely assess patients presented with an acute process of SVC syndrome in the emergency room. Diagnosis can quickly be made by using computed tomography angiography (CTA) or magnetic resonance angiography (MRA) modalities. The underlying cause of the syndrome is the focus of the treatment. Anticoagulation is the basis of the treatment in the case of thrombogenic catheter-associated SVC syndrome. In order to promptly manage symptoms, it was observed that balloon angioplasty with stenting and thrombolytics proved to be beneficial. Herein we are describing a 68-year-old female with past medical history of colon cancer with liver metastasis on chemotherapy via port, presented to the emergency room with acute shortness of breath and facial and neck swelling, and was found to have acute superior vena cava syndrome.
\end{abstract}

Keywords: Superior vena cava syndrome; Balloon angioplasty with stent; Thrombolysis; Anticoagulation

\section{Introduction}

When there is obstruction or direct compression of the superior

Manuscript submitted March 31, 2020, accepted April 7, 2020

aDepartment of Medicine, Richmond University Medical Center, Staten Island, NY 10310, USA

bDepartment of Medicine, Zucker School of Medicine/Northwell Health at Mather Hospital, Port Jafferson, NY 11777, USA

'Department of Medicine/Pulmonary-Critical, Richmond University Medical Center, Staten Island, NY 10310, USA

${ }^{\mathrm{d}}$ Corresponding Author: Rana Al-Zakhari, Department of Medicine, Richmond University Medical Center, Staten Island, NY 10310, USA.

Email: rana_alzakhari@yahoo.com

doi: https://doi.org/10.14740/jmc3461 vena cava (SVC), the SVC syndrome occurs. Usually, SVC syndrome is associated with a gradual onset of symptoms but it can be life-threatening in more acute cases. Most cases of SVC syndrome can be accounted for by malignancies such as lymphoma, metastatic lesions, small-cell lung cancer and nonsmall cell lung cancer. Other reported causes include infections like syphilis, actinomycosis, tuberculosis and histoplasmosis. Apart from these, it was reported that vascular diseases, thrombosis, stenosis and mediastinal fibrosis also lead to SVC syndrome $[1,2]$.

Recently, it has been observed that catheter-related SVC syndromes are more acute and seen to be associated with pacemaker leads and venous catheters. It was reported that central vein thrombosis with intravascular devices has a prevalence ranging from $5 \%$ to $42 \%$ whereas other reports showed that out of all the patients using these devices, $1-14 \%$ of them go on to develop SVC syndrome. In more acute cases of SVC syndrome, it is necessary to consider causes apart from direct compression by tumors [3].

\section{Case Report}

There was a 68 -year-old female with past medical history of being diagnosed with colon cancer in 2008. Present status was post colon resection with adjuvant chemotherapy. In 2008, the patient had a recurrence involving metastasis to the liver, which was then treated with metastectomy followed by a chemotherapy regimen. The patient was brought into the emergency room presenting with a sudden onset of facial swelling and pain which had begun before she went to sleep that night. Later, she woke up in the middle of the night experiencing bluish facial skin discoloration accompanied with severe shortness of breath. She had undergone the most recent chemotherapy cycle just 4 days ago to treat colon cancer with liver metastasis. The patient complained of wheezing along with lip and neck swelling. It was noted that the patient had an allergy to pollen extract, cats, dogs and several food items. At the time of the symptoms onset, the patient denied any previous exposure to known allergens or experiencing similar episodes. On the right side of her chest, the patient had a port.

The patient was treated in the emergency room with intravenous Benadryl $50 \mathrm{mg}$, famotidine $20 \mathrm{mg}$ and Solumedrol $125 \mathrm{mg}$. She was also administered with an intramuscular in- 


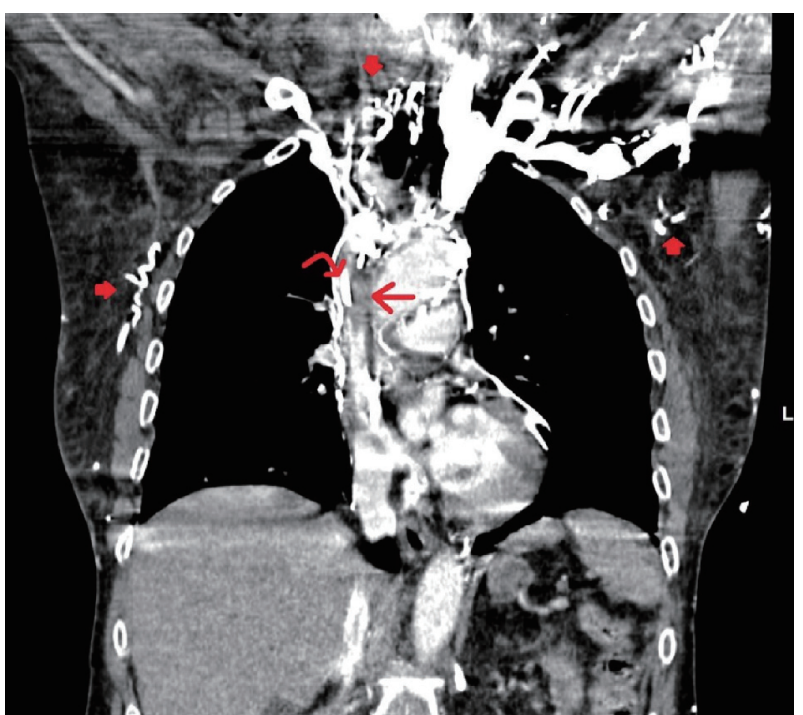

Figure 1. Contrast-enhanced coronal images of the chest in the arterial phase show a hypodense filling defect in the superior vena cava (SVC) surrounded by a thin rim of contrast (straight arrow). A tubular hyperdensity (curved arrow) is seen within the filling defect, consistent with known right port-A catheter. Multiple serpiginous hyperdensities are seen in the thoraco-abdominal wall due to collateral flow (filled arrows). Findings indicate a partially occlusive SVC thrombus surrounding a port catheter with collateral flow.

jection of $0.3 \%$ epinephrine and then placed on a Ventimask of $50 \%$. The patient was placed on bi-level positive airway pressure (BiPAP) machine after the initial treatment failed to show improvement. The patient was treated in the emergency room for anaphylactic reaction for unknown reason. The patient was shown to be awake, oriented and alert upon evaluation. She was observed to be in distress and using her accessory muscles to breath. Her heart rate was 104 beats/min, respiratory rate was 30 breaths/min and the blood pressure was $165 / 100 \mathrm{~mm}$ $\mathrm{Hg}$. The general examination was remarkable for periorbital swelling and facial blush discoloration. Symmetrical swelling on the neck was observed. Lung examination was remarkable for tachypnea and using the accessory muscles was noted, no crackles, stridor or wheezing. Cardiac examination revealed tachycardia, no murmur or gallop. The patient had swollen upper extremities and she was asked to elevate her arms till they touch her face. The patient started complaining of increased pain in her arms and shortness of breath and she soon turned blue.

The laboratory investigations of the patient revealed hemoglobin (Hb) $13 \mathrm{~g} / \mathrm{dL}$, white blood cell (WBC) $29.5 \times 10^{3} / \mu \mathrm{L}$ and platelets $159 \times 10^{3} / \mu \mathrm{L}$. Arterial blood gas analysis showed a $\mathrm{pH}$ of 7.53 , partial pressure of oxygen $\left(\mathrm{PO}_{2}\right)$ of $161 \mathrm{~mm} \mathrm{Hg}$ and bicarbonate $\left(\mathrm{HCO}_{3}\right)$ of $18 \mathrm{mmol} / \mathrm{L}$. Troponin was found to be negative and the basal metabolic panel was observed as unremarkable. Partial prothrombin time of $22.2 \mathrm{~s}$, internal normalized ratio of 1.03 and prothrombin time of $13.4 \mathrm{~s}$ were shown by the coagulation studies. Sinus tachycardia with heart rate of 104 beats/min and no ST elevation or depression was shown in the electrocardiogram (ECG). Except for the internal jugular vein port-A catheter, the chest X-ray came out clear. A chest computed tomography (CT) scan with intravenous contrast was ordered, and the suspicion of thrombus within SVC was raised by the presence of abundant collateral vessels revealed in the report (Fig. 1).

The patient was immediately started on a heparin drip and then moved to the intensive care unit (ICU). Consultations were sought for thrombectomy from the Interventional Radiology and Vascular Surgery. During the procedure, a focal hemodynamically significant stricture of the SVC was revealed by venography, which was adjacent to the tip of the right internal jugular vein port-A catheter. The antegrade blood flow was restored after successfully achieving near complete interval thrombolysis. Minimal residual clot was analyzed by starting the tissue plasminogen activator (TPA) at $1 \mathrm{mg} / \mathrm{h}$. Further TPA therapy was deferred after successful thrombolysis of the focal thrombus was revealed by the venogram 1 day later along with persistent non-occlusive small thrombus in the SVC. The patient's condition improved, and her symptoms resolved. The patient was started on oral anticoagulation. Few days later the patient was discharged home on oral anticoagulation.

\section{Discussion}

The SVC obstruction may be caused by several reasons. Nearby structures like mediastinal masses or enlarged lymph nodes can easily compress the vein due to its location. Catheter-related thrombosis has many associated risk factors. The endothelial wall may be damaged by the central catheter's placement. Blood stasis may also be caused by impeded blood flow in a vein from catheters. The three factors believed to contribute to thrombosis are the components of Virchow's triad including hypercoagulability [4].

The collateral venous return to the heart is developed mainly through the azygos venous system as a result of SVC obstruction. With gradually SVC compression as the result of the tumor compression, the sequelae of the SVC syndrome is developed and minimized by adequate collateral venous return as the SVC obstructions progress gradually. But the presentation may be more life-threatening in a more acute obstruction similar to what is seen with thrombosis. Reports show that it is very rare for SVC syndrome to be caused by colorectal cancer since the only link found has been the rare chance of metastasis to the mediastinal lymph nodes [5]. The most likely etiology of this patient's presentation is from the catheter given the catheter-related risk factors, acute symptoms presented, and the low incidence of SVC syndrome being caused by colorectal cancer [6].

SVC syndrome has some common signs and symptoms which include upper extremity, neck and facial swelling associated with cough, plethora and dyspnea. The "Pemberton's sign" is described as the appearance of flushing, jugular venous distention and facial edema that is aggravated if the patient keeps his arms raised above his head [7]. Some other findings may include hoarseness, dysphagia, stridor, odynophagia, chest pain, changes in mental status, headache, syncope, and periorbital edema. The evaluation of these patients should include diseases like angioedema, constrictive pericarditis and 
congestive heart failure in the differential diagnosis $[4,7,8]$.

Imaging is required for a definitive diagnosis even though it is possible to diagnose SVC syndrome clinically with a detailed history and physical exam. A quick method that is inexpensive without using any radiation or contrast dyes is the ultrasound, but the brachiocephalic and central subclavian veins cannot be visualized due to its limited capabilities. An acoustic shadow caused by the clavicle is responsible for this. It is necessary to direct treatment and monitor how the therapy is progressing with the help of contrast venography, which also helps confirm the SVC syndrome diagnosis. Still, there are many drawbacks with contrast venography. An allergic reaction or nephrotoxicity can be caused by the iodine agents used in contrast venography. During this procedure, cannulation difficulty occurs in the setting of obstruction. The process of venous cannulation was very challenging in our case for contrast venography. In the emergency department, therefore, computed tomography angiography (CTA) and magnetic resonance angiography (MRA) imaging modalities were preferred because they were non-invasive, had minimal complications generally and provided an accurate diagnosis. Evaluating the extent of the obstruction and achieving details similar to venography are possible with these imaging modalities. SVC syndrome can be indicated by seeing the formation of collateral vessels with a sensitivity and specificity of $96 \%$ in CTA and $92 \%$ in MRA [9].

The severity of symptoms and the etiology help decide the type of treatment. Intubation should be carried out immediately if there is a suspicion of airway compromise. Thrombolysis and anticoagulation may also be indicated if thrombosis has been found [9]. With the help of catheter-directed infusion of thrombolytic therapy, good results are being achieved from endovascular treatment and fibrinolytic therapy [10]. The treatments of lower extremity deep vein thrombosis or pulmonary embolism should dictate dosing because the treatment of upper extremity thrombosis has not been evaluated by any randomized controlled studies. Parenteral anticoagulants like heparin should be used during initial therapy followed by warfarin or low molecular weight heparin (LMWH). No studies have been carried out to compare the differences between LMWH and warfarin in upper extremity thromboses [10]. Cases where thrombus or stenosis via catheter is the cause behind SVC syndrome, symptomatic relief can be provided by using balloon angioplasty with stenting as the first line of therapy [4].

Radiation and chemotherapy have to be considered when SVC syndrome is caused by malignancy because they will decrease the tumor burden and the symptoms can potentially be resolved. It is preferred to apply chemotherapy for chemosensitive tumors, and once the biopsy has been performed only then should radiotherapy be done. The traditional methods of elevating the head of the bed, furosemide and dexamethasone have not displayed any benefits in clinical trials [10].

\section{Conclusions}

SVC syndrome is known to be caused by tumor compression. Given the increased use of indwelling catheters and pacemakers, acute SVS syndrome has become reported in few cases.
Given the fact that SVC syndrome can be fatal, early diagnosis and treatment should not be delayed.

\section{Acknowledgments}

We would like to thank Dr. Soham Patel in the Department of Radiology for helping collect the imaging studies.

\section{Financial Disclosure}

None to declare.

\section{Conflict of Interest}

The authors report no conflict of interest.

\section{Informed Consent}

Not applicable.

\section{Author Contributions}

Rana Al-Zakhari collected data, guided the literature search, and wrote the manuscript. Basma Ataallah and Harith Alataby helped collecting data and writing the article. Jay Nfonoyim reviewed and supervised the study.

\section{Data Availability}

The authors declare that data supporting the findings of this study are available within the article.

\section{References}

1. Wilson LD, Detterbeck FC, Yahalom J. Clinical practice. Superior vena cava syndrome with malignant causes. N Engl J Med. 2007;356(18):1862-1869.

2. Rice TW, Rodriguez RM, Light RW. The superior vena cava syndrome: clinical characteristics and evolving etiology. Medicine (Baltimore). 2006;85(1):37-42.

3. Zisis C, Skevis K, Kefaloyannis EM, Avgoustou K, Bellenis I. Mediastinal tuberculous lymphadenitis presenting as superior vena cava syndrome. J Thorac Cardiovasc Surg. 2006;131(6):e11-12.

4. Warren P, Burke C. Endovascular management of chronic upper extremity deep vein thrombosis and superior vena cava syndrome. Semin Intervent Radiol. 2011;28(1):3238.

5. Yavas O, Eren O, Artac M, Boruban C, Genc M. A case of superior vena cava syndrome caused by colon cancer treated with bevacizumab combination chemotherapy. 
Turkish Journal of Cancer. 2009;39(3):115-116.

6. Khalili B, Boggs PB, Bahna SL, Marsala AJ, Tapolyai M, Bryn RO. Superior vena cava syndrome: A masquerader of anaphylaxis. J Allergy Clin Immunol. 2007;119(Supple 1):S29.

7. Shaikh I, Berg K, Kman N. Thrombogenic catheter-associated superior vena cava syndrome. Case Rep Emerg Med. 2013;2013:793054.

8. Koetters KT. Superior vena cava syndrome. J Emerg Nurs. 2012;38(2):135-138; quiz 199.
9. Crispo MM, Fidalgo G, Fix ML, Higgins GL, 3rd. A case of superior vena cava syndrome demonstrating pemberton sign. J Emerg Med. 2012;43(6):1079-1080.

10. Kearon C, Akl EA, Comerota AJ, Prandoni P, Bounameaux H, Goldhaber SZ, Nelson ME, et al. Antithrombotic therapy for VTE disease: Antithrombotic Therapy and Prevention of Thrombosis, 9th ed: American College of Chest Physicians Evidence-Based Clinical Practice Guidelines. Chest. 2012;141(2 Suppl):e419Se496S. 